\title{
An Inverse Mathematical Approach for Thermal Stresses in a Solid Sphere
}

\author{
A.A.Bhave ${ }^{1}$, K.C.Deshmukh ${ }^{1}$, V.S.Kulkarni ${ }^{2, *}$ \\ ${ }^{1}$ Department of Mathematics, RTM Nagpur University, Nagpur-440010, Maharashtra, India \\ ${ }^{2}$ Department of Mathematics, University of Mumbai, Mumbai-400098, Maharashtra, India \\ E-mail: ${ }^{1}$ ashagold@gmail.com, ${ }^{1}$ kcdeshmukh2000@ rediffmail.com, ${ }^{3}$ vinayakskulkarni1@ @ rediffmail.com
}

Received 01 April 2015, Revised 18 July 2015, Accepted 24 July 2015

\begin{abstract}
The present work deals with the determination of unknown temperature and thermal stresses in a solid sphere. A solid sphere is subjected to arbitrary known interior temperature under steady state. The Legendre's transform are used for heat transfer analysis to determine temperature change within solid sphere. The solution of Navier's equation in terms of Goodier's thermoelastic displacement potential and the Boussinesq's harmonic function for spherical co-ordinate system have been used for thermal stress analysis. The results for temperature change, displacement and stresses have been computed numerically and illustrated graphically.
\end{abstract}

Keywords: Heat transfer analysis; steady state; Inverse; Thermal Stress analysis.

AMS: 35G30, 35K05, 44A15, 74A10, 74F05.

\section{Introduction}

The inverse heat conduction problem is one of the most frequently encountered problems by scientists. The wide varieties of problems that are covered under conduction also make it one of the most researched and thought about problems in the field of engineering and technology. This kind of problems can be solved by various methods. These inverse problems consist of determination of unknown temperature, heat flux, displacement and thermal stress functions of solids when the conditions of temperature and displacement and stress are known at the some points of the solid under consideration.

Cialkowski et.al. [1] investigated method of solution of an inverse problem of one dimensional temperature and stresses field for a sphere. Haghighi et al. [2] studied the two dimensional inverse transient heat conduction problem of functionally graded materials. Huang et al. [3] solved a three dimensional transient inverse heat conduction problem by using the conjugate gradient method. Choulli et al. [4] developed a Laplace transform method to solve an inverse problem associated with a boundary value problem in a domain with a simple geometry. Taler et al. [5] present a method where the interior temperature measurements were converted into local instantaneous heat transfer coefficient by solving he inverse heat conduction problem. Noda et al. [6] discussed an analytical method for inverse problem of three dimensional transient thermoelasticity in a transversely isotropic solid by integral transform technique with newly designed potential function and illustrated practical applicability of the method in engineering problem. Mohammadiun et al. [7] has investigated the problem of estimation of time dependent heat flux on a spherical cap using the temperature measurement at one point inside the medium. Kulkarni et al. [8] solved the inverse problem of thermal stresses in a thick circular plate.
This paper deals with the realistic problem of inverse quasi-static steady state thermal stresses in a solid sphere, which is subjected to arbitrary interior temperature. The unknown temperature and thermal stresses on a solid sphere is required to be determined.

\section{Formulation of the Problem}

Following Noda et al. [1], Navier's equation of thermoelasticity for axisymmetric problems are expressed as

$$
\begin{aligned}
& (\lambda+2 \mu) \frac{\partial e}{\partial r}-\frac{2 \mu}{r \sin \theta} \frac{\partial\left(\omega_{\phi} \sin \theta\right)}{\partial \theta}-\beta \frac{\partial \tau}{\partial r}+F_{r}=0 \\
& (\lambda+2 \mu) \frac{1}{r} \frac{\partial e}{\partial \theta}+\frac{2 \mu}{r} \frac{\partial\left(r \omega_{\phi}\right)}{\partial r}-\beta \frac{1}{r} \frac{\partial \tau}{\partial \theta}+F_{\theta}=0 \\
& \text { where } \quad \omega_{\phi}=\frac{1}{2 r}\left[\frac{\partial\left(r \mu_{\theta}\right)}{\partial r}-\frac{\partial \mu_{r}}{\partial \theta}\right]
\end{aligned}
$$

The solution of the Navier's equation without the body force for axisymmetric problems in the spherical coordinate system are expressed, in terms of Goodier's thermoelastic displacement potential $\Phi$ and the Boussinesq harmonic functions $\varphi$ and $\psi$ as

$$
\begin{aligned}
& \mu_{r}=\frac{\partial \Phi}{\partial r}+\frac{\partial \varphi}{\partial r}+r \cos \theta \frac{\partial \psi}{\partial r}-(3-4 v) \psi \cos \theta \\
& \mu_{\theta}=\frac{1}{r} \frac{\partial \Phi}{\partial \theta}+\frac{1}{r} \frac{\partial \varphi}{\partial \theta}+\cos \theta \frac{\partial \psi}{\partial \theta}+(3-4 v) \psi \sin \theta
\end{aligned}
$$

where the three functions must satisfy the equations

$\nabla^{2} \Phi=K \tau$

$\nabla^{2} \varphi=0$ 


$$
\begin{aligned}
& \nabla^{2} \psi=0 \\
& \nabla^{2}=\frac{\partial^{2}}{\partial r^{2}}+\frac{2}{r} \frac{\partial}{\partial r}+\frac{1}{r^{2}} \frac{\partial^{2}}{\partial \theta^{2}}+\frac{1}{r^{2}} \cot \theta \frac{\partial}{\partial \theta} \\
& K=\frac{1+v}{1-v} \alpha
\end{aligned}
$$

The stress components in the spherical coordinate system are represented in terms of three functions $\Phi, \varphi$ and $\psi$ are

$$
\begin{aligned}
& \sigma_{r r}=2 G[ {\left[\frac{\partial^{2} \Phi}{\partial r^{2}}+\frac{\partial^{2} \varphi}{\partial r^{2}}+r \cos \theta \frac{\partial^{2} \psi}{\partial r^{2}}-2(1-v) \cos \theta \frac{\partial \psi}{\partial r}\right.} \\
&\left.+2 v \frac{1}{r} \sin \theta \frac{\partial \psi}{\partial \theta}-K \tau\right] \\
& \sigma_{\theta \theta}=2 G\left[\frac{1}{r} \frac{\partial \Phi}{\partial r}+\frac{1}{r^{2}} \frac{\partial^{2} \Phi}{\partial \theta^{2}}+\frac{1}{r} \frac{\partial \varphi}{\partial r}+\frac{1}{r^{2}} \frac{\partial^{2} \varphi}{\partial \theta^{2}}+(1-2 v) \cos \theta\right. \\
&\left.\times \frac{\partial \psi}{\partial r}+\frac{1}{r} \cos \theta \frac{\partial^{2} \psi}{\partial \theta^{2}}+2(1-v) \frac{1}{r} \sin \theta \frac{\partial \psi}{\partial \theta}-K \tau\right](11) \\
& \sigma_{\phi \phi}=2 G\left[\frac{1}{r} \frac{\partial \Phi}{\partial r}+\cot \theta \frac{1}{r^{2}} \frac{\partial \Phi}{\partial \theta}+\frac{1}{r} \frac{\partial \varphi}{\partial r}+\cot \theta \frac{1}{r^{2}} \frac{\partial \varphi}{\partial \theta}\right. \\
&+(1-2 v) \cos \theta \frac{\partial \psi}{\partial r}+(\cos \theta \cot \theta+2 v \sin \theta) \frac{1}{r} \frac{\partial \psi}{\partial \theta} \\
&-K \tau] \\
& \sigma_{r \theta}= 2 G\left[\frac{\partial^{2}}{\partial r \partial \theta}\left(\frac{\Phi}{r}\right)+\frac{\partial^{2}}{\partial r \partial \theta}\left(\frac{\varphi}{r}\right)+(1-2 v) \sin \theta \frac{\partial \psi}{\partial r}\right. \\
&\left.+\cos \theta \frac{\partial^{2} \psi}{\partial r \partial \theta}-2(1-v) \frac{1}{r} \cos \theta \frac{\partial \psi}{\partial \theta}\right]
\end{aligned}
$$

The mechanical boundary conditions on the traction free surface $r=b$ are

$\sigma_{r r}=0, \sigma_{r \theta}=0$ on $r=b$

Eqs, (1)-(14) constitute the general thermoelastic formulation of two dimensional spherical problem.

\section{Governing Equation of Heat Conduction}

Consider a two-dimensional solid sphere of radius $b$ defined by $0 \leq r \leq b,-1 \leq \mu \leq 1$. The radius $r=\xi$ is kept at temperature $f(\mu)$. Assume the boundary surface of a solid sphere is free from traction. Under these prescribed conditions, the thermal stresses are required to be determined.

A steady state temperature of a sphere satisfies the heat conduction equation,

$$
\begin{aligned}
& \frac{1}{r^{2}} \frac{\partial}{\partial r}\left(r^{2} \frac{\partial T}{\partial r}\right)+\frac{1}{r^{2}} \frac{\partial}{\partial \mu}\left[\left(1-\mu^{2}\right) \frac{\partial T}{\partial \mu}\right]=0 \\
& 0 \leq r \leq b,-1 \leq \mu \leq 1 \text { for } t>0 \\
& \text { with } \quad T=g(\mu) \text { (unknown) at } r=b \\
& T=f(\mu) \text { (known) at } r=\xi, 0 \leq \xi<b \\
& \text { where } T=T(r, \mu)
\end{aligned}
$$

\subsection{The Solution for Temperature Change}

The partial derivative with respect to $\mu$ variable can be removed by means of Legendre transform. Define the Legendre transform and the corresponding inversion formula of the temperature function $T(r, \mu)$ with respect to the variable $\mu$ as

$$
\bar{T}(r, n)=\int_{\mu^{\prime}=-1}^{1} K_{n}\left(\mu^{\prime}\right) T\left(r, \mu^{\prime}\right) d \mu^{\prime}
$$

and

$$
T(r, \mu)=\sum_{n=0}^{\infty} K_{n}(\mu) \bar{T}(r, n)
$$

where

$$
K_{n}(\mu)=\sqrt{\frac{2 n+1}{2}} P_{n}(\mu) \quad n=0,1,2,3, \cdots
$$

By applying Legendre's transform defined in the Eq. (19) to governing heat conduction equation (15) along with the boundary condition as defined in Eq. (17), one obtain

$\frac{d}{d r}\left(r^{2} \frac{d \bar{T}}{d r}\right)-n(n+1) \bar{T}=0$ in $0 \leq r \leq b$

with $\quad \bar{T}=\bar{f}(n)$ at $r=\xi$

On solving above equation (22), one obtain

$\bar{T}=C_{1} r^{n}+C_{2} r^{-(1+n)}$

The solution $r^{-(1+n)}$ is excluded because the temperature should remain finite at $r=0$.

$\bar{T}=C_{1} r^{n}$

Using boundary condition prescribed in Eq. (23), one obtain

$$
C_{1}=\frac{\bar{f}(n)}{\xi^{n}}
$$

Hence Eq (25) gives

$$
\bar{T}=\bar{f}(n)\left(\frac{r}{\xi}\right)^{n}
$$

By applying inverse Legendre's transform as defined in Eq. (20), one obtain

$$
\begin{aligned}
& T(r, \mu)=\sum_{n=0}^{\infty} K_{n}(\mu)\left(\frac{r}{\xi}\right)^{n} \int_{\mu^{\prime}=-1}^{1} f\left(\mu^{\prime}\right) K_{n}\left(\mu^{\prime}\right) d \mu^{\prime} \\
& T(r, \mu)=\sum_{n=0}^{\infty} \sqrt{\frac{2 n+1}{2}} P_{n}(\mu)\left(\frac{r}{\xi}\right)^{n} \int_{\mu^{\prime}=-1}^{1} f\left(\mu^{\prime}\right) K_{n}\left(\mu^{\prime}\right) d \mu^{\prime}
\end{aligned}
$$

The unknown temperature $g(\mu)$ can be obtained by substituting $r=b$ in Eq. (29) as

$$
g(\mu)=\sum_{n=0}^{\infty} \sqrt{\frac{2 n+1}{2}} P_{n}(\mu)\left(\frac{b}{\xi}\right)^{n} \int_{\mu^{\prime}=-1}^{1} f\left(\mu^{\prime}\right) K_{n}\left(\mu^{\prime}\right) d \mu^{\prime}
$$

Using Eq. (28), under steady state temperature condition $\tau=T$

$\tau=\sum_{n=0}^{\infty} A_{n} r^{n} P_{n}(\mu)$

where

$A_{n}=\sqrt{\frac{2 n+1}{2}} \frac{1}{\xi^{n}} \int_{\mu^{\prime}=-1}^{1} f\left(\mu^{\prime}\right) K_{n}\left(\mu^{\prime}\right) d \mu^{\prime}$ 


\subsection{The Goodier's thermoelastic displacement potential}

$\Phi$

The Goodier's thermoelastic displacement potential $\Phi$ satisfies the equation

$$
\nabla^{2} \Phi=K \tau
$$

Using value of temperature difference from Eq. (31), one obtain

$$
\frac{\partial^{2} \Phi}{\partial r^{2}}+\frac{1}{r} \frac{\partial \Phi}{\partial r}=K\left[\sum_{n=0}^{\infty} A_{n} r^{n} P_{n}(\mu)\right]
$$

On solving, one obtains the Goodier's thermoelastic displacement potential function $\Phi$ as

$$
\Phi=K\left[\sum_{n=0}^{\infty} \frac{1}{(n+3)(n+2)} A_{n} r^{n+2} P_{n}(\mu)\right]
$$

\subsection{The First Component of Displacement and Thermal \\ Stresses}

The first component of displacement and thermal stress with respect to Goodier's thermoelastic displacement potential function $\Phi$ can be obtained as

$$
\begin{aligned}
& \bar{\mu}_{r}=\frac{\partial \Phi}{\partial r} \\
& \bar{\mu}_{r}=K\left[\sum_{n=0}^{\infty} \frac{1}{(n+3)} A_{n} r^{n+1} P_{n}(\mu)\right] \\
& \bar{\mu}_{\theta}=\frac{1}{r} \frac{\partial \Phi}{\partial \theta} \\
& \bar{\mu}_{\theta}=-K\left(1-\mu^{2}\right)^{\frac{1}{2}} \sum_{n=1}^{\infty} \frac{1}{(n+3)(n+2)} A_{n} r^{n+1} \frac{n+1}{1-\mu^{2}} \\
& \times\left[\mu P_{n}(\mu)-P_{n+1}(\mu)\right] \\
& \bar{\sigma}_{r r}=2 G\left[\frac{\partial^{2} \Phi}{\partial r^{2}}-K \tau\right] \\
& \bar{\sigma}_{r r}=2 G K\left[\sum_{n=0}^{\infty} \frac{-2}{(n+3)} A_{n} r^{n} P_{n}(\mu)\right] \\
& \bar{\sigma}_{\theta \theta}=2 G\left[\frac{1}{r} \frac{\partial \Phi}{\partial r}+\frac{1}{r^{2}} \frac{\partial^{2} \Phi}{\partial \theta^{2}}-K \tau\right] \\
& \bar{\sigma}_{\theta \theta}=2 G K \sum_{n=0}^{\infty}\left[\frac{(-n-2)}{(n+3)} A_{n} r^{n} P_{n}(\mu)+\frac{(n+1)}{(n+3)(n+2)} A_{n} r^{n}\right. \\
& \left.\times\left(\frac{\mu}{1-\mu^{2}}\left[\mu P_{n}(\mu)-P_{n+1}(\mu)\right]\right)\right] \\
& \bar{\sigma}_{\phi \phi}=2 G\left[\frac{1}{r} \frac{\partial \Phi}{\partial r}+\cot \theta \frac{1}{r^{2}} \frac{\partial \Phi}{\partial \theta}-K \tau\right] \\
& \bar{\sigma}_{\phi \phi}=2 G K \sum_{n=0}^{\infty}\left(A_{n} r^{n}\right)\left[\frac{(-n-2)}{(n+3)} \mu^{n}-\frac{(n+1)}{(n+3)(n+2)} \mu\right. \\
& \left.\times \frac{\left[\mu P_{n}(\mu)-P_{n+1}(\mu)\right]}{\left(1-\mu^{2}\right)}\right] \\
& \bar{\sigma}_{r \theta}=2 G\left[\frac{\partial^{2}}{\partial r \partial \theta}\left(\frac{\Phi}{r}\right)\right] \\
& \bar{\sigma}_{r \theta}=-2 G K\left(1-\mu^{2}\right)^{\frac{1}{2}}\left[\sum_{n=1}^{\infty} \frac{(n+1)^{2}}{(n+3)(n+2)} A_{n} r^{n} \frac{1}{1-\mu^{2}}\right.
\end{aligned}
$$

$$
\left.\left[\mu P_{n}(\mu)-P_{n+1}(\mu)\right]\right]
$$

\subsection{The Solution of Laplace Equation}

The solution of Laplace equation satisfying conditions given in Eqs. (6) and (7) are

$\varphi=\sum_{n=0}^{\infty}\left\{\left[C_{1, n}-(n-4+4 v) D_{1, n-2}\right] r^{n}\right\} P_{n}(\cos \theta)$

$\psi=\sum_{n=0}^{\infty}\left[(2 n+1) D_{1, n-1} r^{n}\right] P_{n}(\cos \theta)$

\subsection{The Second Component of Displacement and} Thermal Stresses

$\overline{\bar{\mu}}_{r}=\frac{\partial \varphi}{\partial r}+\mu\left[r \frac{\partial \psi}{\partial r}-(3-4 v) \psi\right]$

$$
\begin{aligned}
\overline{\bar{\mu}}_{r}= & \sum_{n=0}^{\infty}\left\{\left[C_{1, n}-(n-4+4 v) D_{1, n-2}\right] n r^{n-1}+\left[(2 n+1) D_{1, n-1}\right]\right. \\
& \left.\times r^{n} \mu[n-(3-4 v)] P_{n}(\mu)\right\} \\
\overline{\bar{\mu}}_{\theta}= & -\left(1-\mu^{2}\right)^{1 / 2}\left[\frac{1}{r} \frac{\partial \varphi}{\partial \theta}+\mu \frac{\partial \psi}{\partial \theta}-(3-4 v) \psi \sin \theta\right]
\end{aligned}
$$

$$
\begin{aligned}
\overline{\bar{\mu}}_{\theta}=-(1 & \left.-\mu^{2}\right)^{1 / 2} \sum_{n=1}^{\infty}\left\{\left[C_{1, n}-(n-4+4 v) D_{1, n-2}\right] n r^{n-1}\right. \\
& \times \frac{1}{1-\mu^{2}}\left[P_{n-1}(\mu)-\mu P_{n}(\mu)\right]+\left[(2 n+1) D_{1, n-1}\right] r^{n} \\
& \left.\times\left(\left[\frac{n}{1-\mu^{2}}\left(P_{n-1}(\mu)-\mu P_{n}(\mu)\right)\right]-(3-4 v) P_{n}(\mu)\right)\right\}
\end{aligned}
$$

$$
\overline{\bar{\sigma}}_{r r}=2 G\left[\frac{\partial^{2} \varphi}{\partial r^{2}}+\mu r \frac{\partial^{2} \psi}{\partial r^{2}}-2(1-v) \mu \frac{\partial \psi}{\partial r}-2 v \frac{1}{r}\left(1-\mu^{2}\right) \frac{\partial \psi}{\partial \theta}\right]
$$

$$
\begin{aligned}
\overline{\bar{\sigma}}_{r r}= & 2 G \sum_{n=0}^{\infty}\left\{\left[C_{1, n}-(n-4+4 v) D_{1, n-2}\right] n(n-1) r^{n-2} P_{n}(\mu)\right. \\
+ & {\left[(2 n+1) D_{1, n-1}\right] r^{n-1}\left[n \mu[(n-1)-2(1-v)] P_{n}(\mu)\right.} \\
& \left.\left.-2 n v\left[\mu P_{n}(\mu)-P_{n+1}(\mu)\right]\right]\right\} \\
\overline{\bar{\sigma}}_{\theta \theta}= & 2 G\left\{\frac{1}{r} \frac{\partial \varphi}{\partial r}+\frac{1}{r^{2}} \frac{\partial^{2} \varphi}{\partial \theta^{2}}+(1-2 v) \cos \theta \frac{\partial \psi}{\partial r}\right. \\
& \left.+\frac{1}{r} \cos \theta \frac{\partial^{2} \psi}{\partial \theta^{2}}+2(1-v) \frac{1}{r} \sin \theta \frac{\partial \psi}{\partial \theta}\right\} \\
\overline{\bar{\sigma}}_{\theta \theta}= & 2 G \sum_{n=0}^{\infty}\left\{[ C _ { 1 , n } - ( n - 4 + 4 v ) D _ { 1 , n - 2 } ] r ^ { n - 2 } \left[\frac{-n \mu}{1-\mu^{2}} P_{n-1}(\mu)\right.\right. \\
& \left.+\frac{n \mu^{2}}{1-\mu^{2}} P_{n}(\mu)+n \mu^{2} P_{n}(\mu)+2 \mu^{2} P_{n}(\mu)-2 \mu P_{n+1}(\mu)\right] \\
+ & {\left[(2 n+1) D_{1, n-1}\right] r^{n-1}\left[\frac{-n \mu^{2}}{1-\mu^{2}} P_{n-1}(\mu)+\frac{n \mu^{3}}{1-\mu^{2}} P_{n}(\mu)\right.} \\
+ & n \mu^{3} P_{n}(\mu)+2 \mu^{3} P_{n}(\mu)-2 \mu^{2} P_{n+1}(\mu)-2 n P_{n-1}(\mu)
\end{aligned}
$$




$$
\begin{aligned}
\overline{\bar{\sigma}}_{\phi \phi}= & 2 G\left\{\frac{1}{r} \frac{\partial \varphi}{\partial r}+\cot \theta \frac{1}{r^{2}} \frac{\partial \varphi}{\partial \theta}+(1-2 v) \cos \theta \frac{\partial \psi}{\partial r}\right. \\
& \left.+[\cos \theta \cot \theta+2 v \sin \theta] \frac{1}{r} \frac{\partial \psi}{\partial \theta}\right\} \\
\overline{\bar{\sigma}}_{\phi \phi}= & 2 G \sum_{n=0}^{\infty}\left\{[ C _ { 1 , n } - ( n - 4 + 4 v ) D _ { 1 , n - 2 } ] r ^ { n - 2 } \left[n P_{n}(\mu)\right.\right. \\
- & \left.\frac{n \mu}{1-\mu^{2}} P_{n-1}(\mu)+\frac{n \mu^{2}}{1-\mu^{2}} P_{n}(\mu)\right]+\left[(2 n+1) D_{1, n-1}\right] r^{n-1} \\
& \times\left[n \mu P_{n}(\mu)+2 n v \mu P_{n}(\mu)-\frac{2 n v}{1-\mu^{2}} P_{n-1}(\mu)\right. \\
& +\frac{2 n v}{1-\mu^{2}} \mu P_{n}(\mu)-n \mu^{2} P_{n-1}(\mu)+2 v \mu^{2} n P_{n-1}(\mu) \\
& \left.\left.-\frac{n \mu^{3}}{1-\mu^{2}} P_{n}(\mu)-2 v \frac{n \mu^{3}}{1-\mu^{2}} P_{n}(\mu)\right]\right\} \\
+(1- & \left.\left.2 v) n P_{n}(\mu)\right]\right\} \\
& \times \frac{n(n-1)}{1-\mu^{2}}\left[P_{n-1}(\mu)-\mu P_{n}(\mu)\right]+\left[(2 n+1) D_{1, n-1}\right] r^{n-1} \\
& -2(1-v) \frac{\partial^{2}}{r}\left(\frac{\varphi}{\partial r}\right)+(1-2 v) \sin \theta \frac{\partial \psi}{\partial r}+\cos \theta \frac{\partial^{2} \psi}{\partial r \partial \theta} \\
\overline{\bar{\sigma}}_{r \theta}= & 2 G\left(1-\mu^{2}\right)^{1 / 2} \sum_{n-1}^{\infty}\left\{-\left[C_{1, n}-(n-4+4 v) D_{1, n-2}\right] r^{n-2}\right.
\end{aligned}
$$

\subsection{The Displaceent and thermal Stresses}

The displacements and thermal stresses defined in Eqs.

(3), (4) and (10) to (14) can be obtain by adding Eqs.

(37),(39), (41),(43), (45),(47) and (51),(53),(55),(57),(59),

(61), respectively as

$$
\begin{aligned}
& \mu_{r}=\bar{\mu}_{r}+\overline{\bar{\mu}}_{r} \\
& \mu_{\theta}=\bar{\mu}_{\theta}+\overline{\bar{\mu}}_{\theta} \\
& \sigma_{r r}=\bar{\sigma}_{r r}+\overline{\bar{\sigma}}_{r r} \\
& \sigma_{\theta \theta}=\bar{\sigma}_{\theta \theta}+\overline{\bar{\sigma}}_{\theta \theta} \\
& \sigma_{\phi \phi}=\bar{\sigma}_{\phi \phi}+\overline{\bar{\sigma}}_{\phi \phi} \\
& \sigma_{r \theta}=\bar{\sigma}_{r \theta}+\overline{\bar{\sigma}}_{r \theta}
\end{aligned}
$$

The unknowns used in Eqs. (48) and (49), can be determined by solving equilibrium Eq. (14) as

$$
C_{1, n}=\frac{K}{\Delta_{n}} \frac{(2 n+1)}{n-1} \frac{1}{n+3} b^{2} A_{n}
$$

$D_{1, n-2}=-\frac{K}{\Delta_{n}} \frac{2 n+1}{n-1} \frac{1}{n+3} b^{2} A_{n}$

$D_{1, n-1}=-\frac{K}{\Delta_{n}} \frac{1}{n+3} \frac{b}{\mu} A_{n}$

where $\Delta_{n}=n v(2 n+1)$

\section{Special Case and Numerical Calculations}

The mathematical model is constructed by setting $f(\mu)=100\left(1-\mu^{2}\right)^{\frac{1}{2}}$

where $\mu=\cos \theta$,

$f(\mu)=100 \sin \theta$.

The known interior temperature is an application of sinusoidal heat flux at $r=\xi, 0 \leq \xi<b$. For which arbitrary coefficient of temperature is given by using Eq. (31) as

$A_{n}=(2 n+1) \frac{50}{b^{n}}\left(\overline{\frac{\left(\frac{n+1}{2}\right.}{\left(\frac{n}{2}\right) !}}\right)^{2}$

The numerical calculations have been carried out for an iron (pure) solid sphere with the following material properties and dimensions:

$$
\begin{aligned}
& \text { Poisson ratio } v=0.35 \\
& \text { Radius of Sphere } b=1 m \\
& K=\frac{1.35}{0.65}(20.39)\left(10^{-6}\right) \\
& G=0.5 \\
& \mu=0.5
\end{aligned}
$$$$
\text { Thermal diffusivity } \alpha=20.34 \times 10^{-6}\left(\mathrm{~m}^{2} \mathrm{~s}^{-1}\right)
$$

In two dimensional axisymmetric problem of a spherical body, it is assumed that the body is deformed symmetrically with respect to the coordinate axis $\mathrm{z}$. The numerical calculations have been carried out with the help of computational mathematical software Mathcad-2000 and the graphs are plotted with the help of excel (MS office 2000).

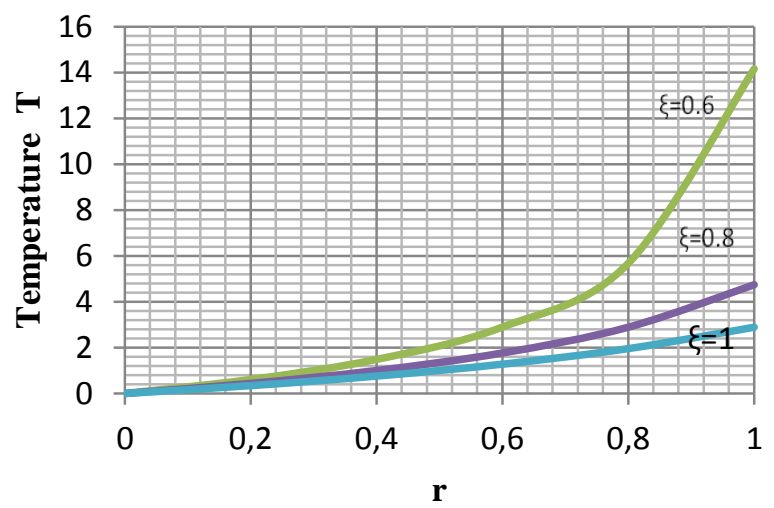

Fig.1. The temperature $T$ along radial direction. 


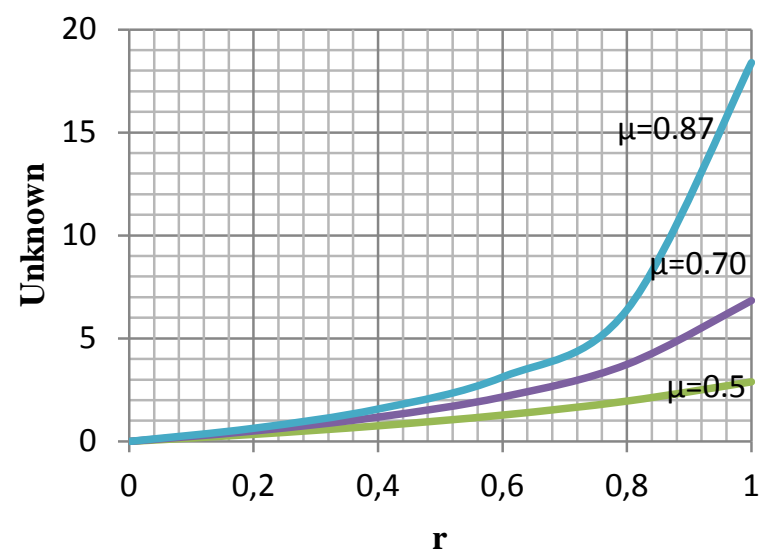

Fig.2.The unknown g along radial direction.

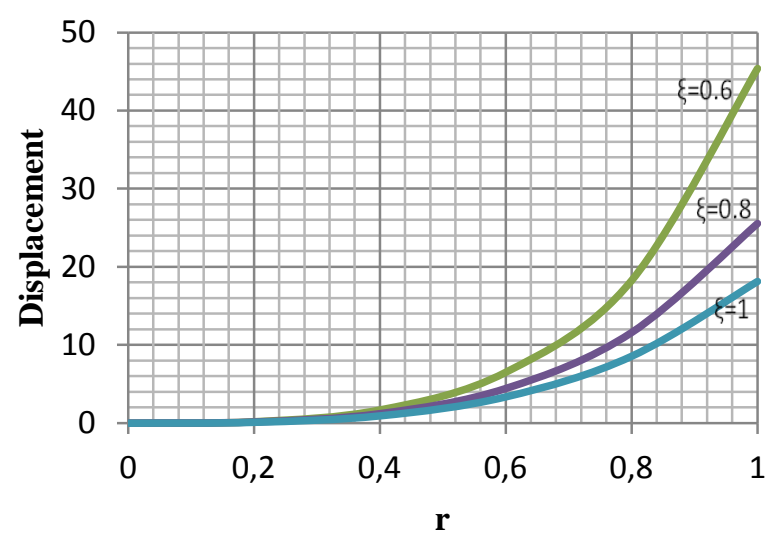

Fig.3.The displacement $\Phi$ along radial direction.

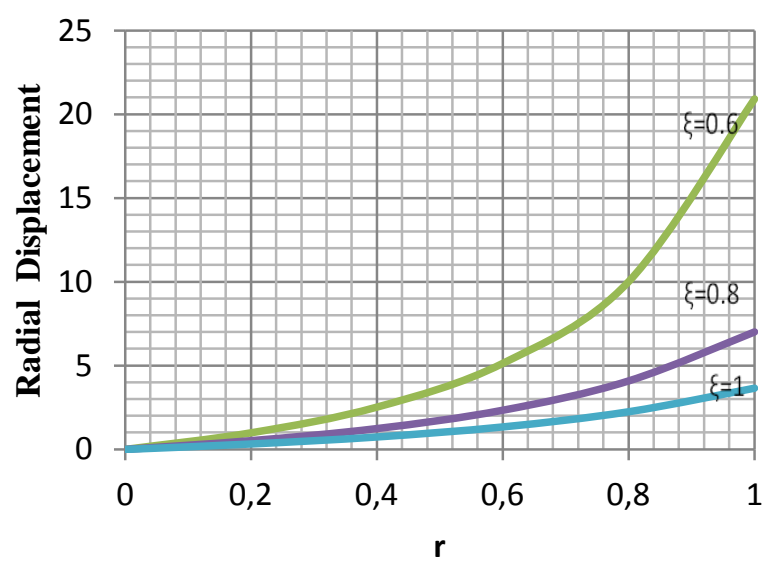

Fig.4.The radial displacement $\mu_{r}$ along radial direction.

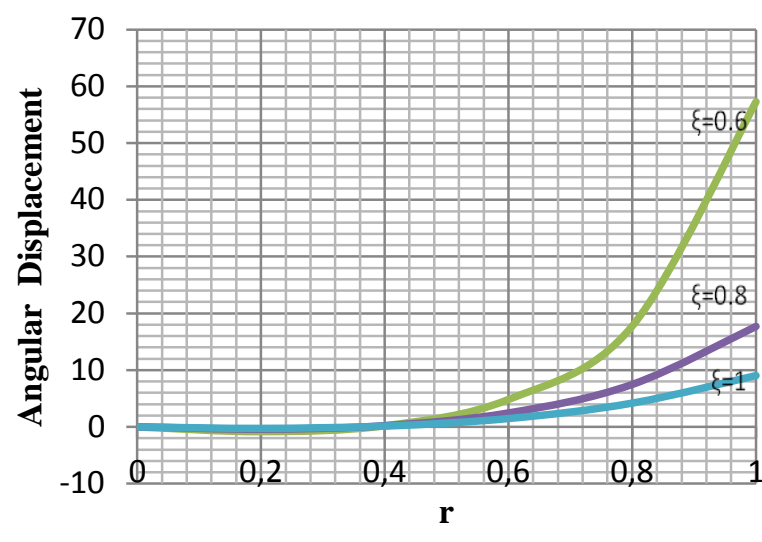

Fig.5.The angular displacement $\mu_{\theta}$ along radial direction.

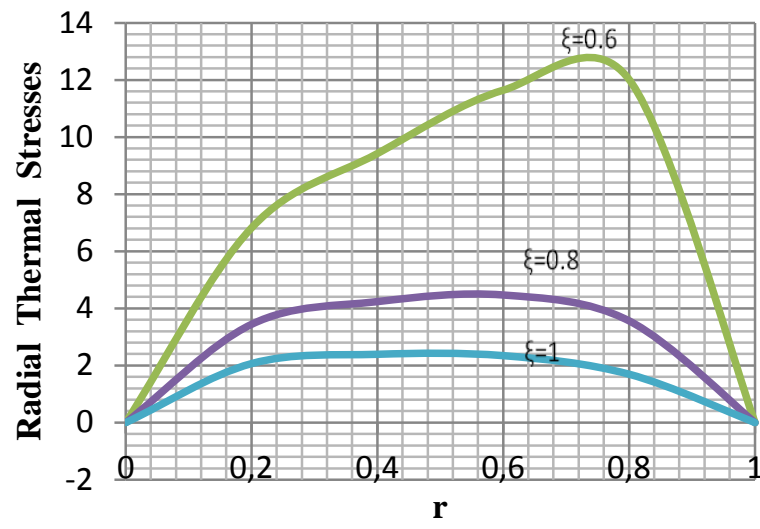

Fig.6. The radial thermal stresses $\sigma_{r r}$ along radial direction.

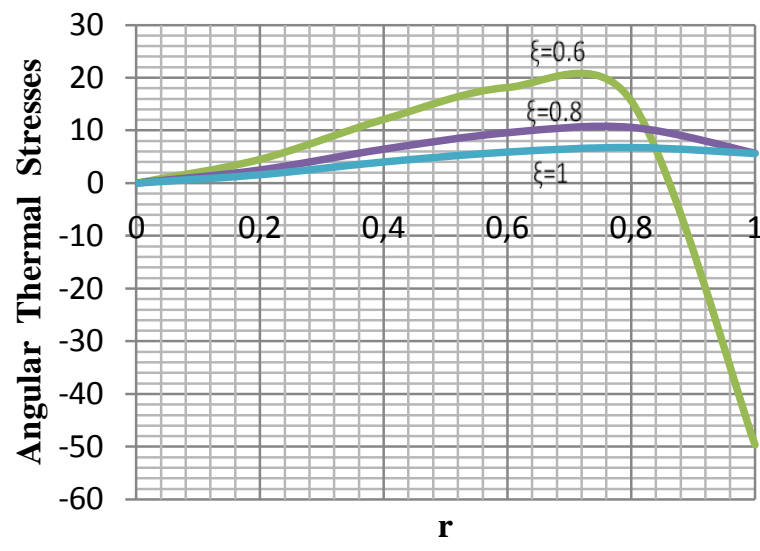

Fig.7. The angular thermal stresses $\sigma_{\theta \theta}$ along radial direction. 


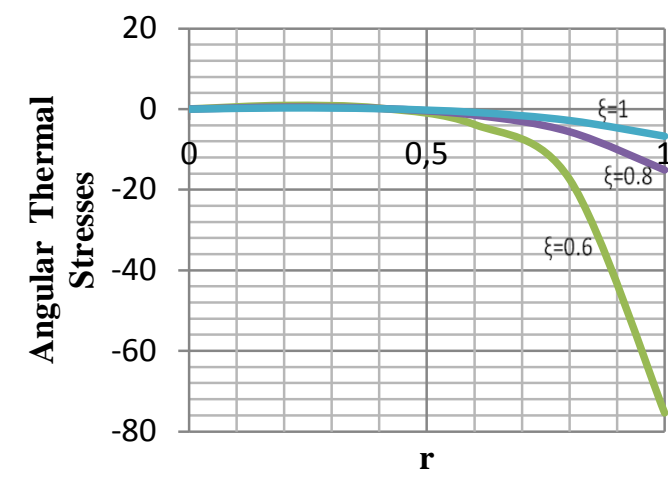

Fig.8.The angular thermal stresses $\sigma_{\phi \phi}$ along radial direction.

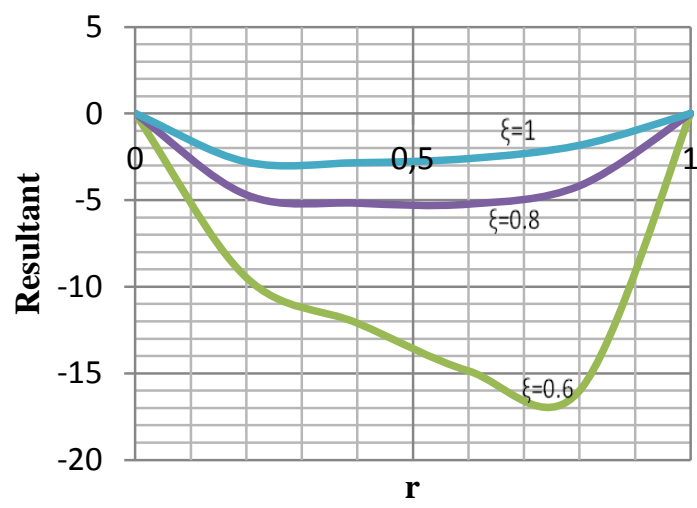

Fig.9.The resultant $\sigma_{r \theta}$ along radial direction.

\section{Results and Discussion}

From Figures 1 and 2 , it is observed that the temperature $T$ increases from center to the outer spherical surface. The unknown temperature $g(\mu)$ shows variation on the outer spherical boundary. From Figures 3, 4 and 5, the displacement $\Phi$ the radial displacement $\mu_{r}$ and angular displacement $\mu_{\theta}$ increases as $r$ increases. Displacement of solid sphere can be observed on outer spherical boundary. From Figure 6, it is observed that the radial thermal stresses develop tensile stresses. The stress function $\sigma_{r r}$ is zero at the outer spherical boundary $(r=1)$ and at the center of sphere. From Figures 7 and 8, the angular thermal stresses show compressive stress on outer spherical surface. From Figure 9 , it is seen that that the resultant function $\sigma_{r \theta}$ is tending to zero at the outer spherical boundary $(r=1)$. It develops compressive stresses in radial direction.

\section{Conclusions}

Equations are derived for temperature, displacement, and thermal stresses for a solid sphere which is subjected to arbitrary known interior temperature. As a special case mathematical model is constructed for pure iron solid sphere.

In order to study the characteristic of temperature variation, displacement and thermal stresses within solid sphere, the uncoupled thermoelasticity with infinite wave propagation in the form of heat are discussed. The Legendre's transform are used for heat transfer analysis to determine temperature change within solid sphere. The solution of Navier's equation in terms of Goodier's Vol. 18 (No. 3) / 211 thermoelastic displacement potential and the Boussinesq's harmonic function for spherical co-ordinate system have been used for thermal stress analysis. As a special case, sinusoidal heat flux is considered and performed numerical calculation for special case and illustrated these variations graphically along radial direction. The displacement and thermal stresses developed on the outer spherical boundary can create expansion of solid sphere. Any particular case can be analyzed by these general solutions obtained.

$\begin{array}{ll}\text { Nomenclature } & \\ r & \text { Radius of sphere } \\ T(r, \theta) & \text { Temperature at any point } \\ \tau & \text { Temperature change } \\ \alpha & \text { Thermal diffusivity of material } \\ \mathrm{K} & \text { Restraint coefficient } \\ F_{r} \text { and } F_{\theta} & \text { Forces in radial and angular direction } \\ \Phi & \text { Goodier's thermoelastic potential } \\ \varphi \text { and } \psi & \text { Boussinesq harmonic function } \\ G & \text { Shear modulus } \\ v & \text { Poisson's ratio } \\ \mu_{r} & \text { Radial displacement } \\ \mu_{\theta} & \text { Angular displacement } \\ \sigma_{r r}, \sigma_{r \theta}, \sigma_{\theta \theta}, \sigma_{\phi \phi} & \text { Components of stress function } \\ \mu, \lambda & \text { Lame constant } \\ P_{n}(x) & \text { Legendre polynomials }\end{array}$

\section{References}

[1] Cialkowski N.J., Grysa K.W., "On a certain Inverse problem of temperature and thermal Stresses fields", Acta Mechanica, 36, 169-185, 1981.

[2] Haghighi G., Eghtesad M., Malekzadeh P., Necsulescu D.S., "Two dimensional inverse heat transfer analysis of functionally graded materials in estimating time dependent surface heat flux", Numerical heat transfer, Part A: Applications, 54, 744-762, 2008.

[3] Huang C H., Cheng S.C., "Three dimensional inverse problem of estimating the volumetric heat generation for a composite material", Numerical heat transfer, Part A: Applications, 39, 383-403, 2001.

[4] Choulli M., Zeghal A., "Laplace transform approach for an inverse problem", Transport Theory and Statistical Physics, 24, 1353-1367, 1995.

[5] Taler J., "Theory of transient experimental technique for surface heat transfer", Int. J. Heat Mass Transfer, 42, 1123-1140, 1999.

[6] Noda N., Hetnarski R. ,Tanigawa Y., "Thermal Stresses in spherical bodies", in Thermal Stresses, $2^{\text {nd }}$ edition, New York, Taylor \& Francis, 2003, Chp.7, p.295-341.

[7] Mohammadium M., Rahimi A.B., "Estimation of heat flux using temperature distribution at a point by conjugate gradient method", Int. J. Thermal Sciences, 50, 2443-2450, 2011.

[8] Kulkarni V.S., Deshmukh K.C., "An inverse quasistatic steady state thermal stresses in a thick circular plate", J. Franklin Institute, 345, 29-38, 2008.

[9] Ozisik M.N., "Heat conduction in spherical coordinate system" in Boundary value problems of heat conduction, Scranton, Pennsylvania, International Company, 1968, pp.194-236. 